\title{
Errors in the Total Testing Process in the Clinical Chemistry Laboratory at the University of Gondar Hospital, Northwest Ethiopia
}

\author{
Sintayehu Ambachew ${ }^{1 *}$, Kasaw Adane ${ }^{3}$, Abebaw Worede ${ }^{2}$, Tadele Melak ${ }^{1}$, \\ Daniel Asmelash ${ }^{1}$, Shewaneh Damtie ${ }^{2}$, Habtamu Wondifraw Baynes ${ }^{1}$, Molla \\ Abebe $^{1}$, Belete Biadgo ${ }^{1}$
}

\begin{abstract}
OPEN ACCESS
Citation: Sintayehu Ambachew, Kasaw Adane, Abebaw Worede, Tadele Melak, Daniel Asmelash, Shewaneh Damtie, Habtamu Wondifraw Baynes, Molla Abebe, Belete Biadgo. Errors in the Total Testing Process in the Clinical Chemistry Laboratory at the University of Gondar Hospital, Northwest Ethiopia. Ethiop J Sci.2018;28(2):235.

doi:http://dx.doi.org/10.4314/ejhs.v28i2.15 Received: October 24, 2017

Accepted: November 24, 2017

Published: March 1, 2018

Copyright: (C) 2018 Sintayehu Ambachew, et al. This is an open access article distributed under the terms of the Creative Commons Attribution License, which permits unrestricted use, distribution, and reproduction in any medium, provided the original author and source are credited.

Funding: University of Gondar

Competing Interests: The authors declare that this manuscript was approved by all authors in its form and that no competing interest exists.

Affiliation and Correspondence:

${ }^{1}$ Department of Clinical Chemistry, School of Biomedical and Laboratory

Sciences, College of Medicine and

Health Sciences, University of

Gondar, Gondar, Ethiopia

${ }^{2}$ University of Gondar Hospital

Laboratory, College of Medicine and

Health Sciences, University of

Gondar, Gondar, Ethiopia

${ }^{3}$ Unit of Quality Assurance and

Laboratory Mangement, College of

Medicine and Health Sciences,

University of Gondar, Gondar,

Ethiopia

*Email: sinte.ambachew@gmail.com
\end{abstract}

ABSTRACT

BACKGROUND: Laboratory services have been described as the major processes contributing to safe patient care in the modern healthcare sector. However, occurrences of errors in the overall testing processes impair the clinical decision-making process. Such errors are supposed to be high in resource-poor countries, like Ethiopia. The objective of this study was to assess errors in the total testing process in the Clinical Chemistry laboratory of the University of Gondar Hospital, Northwest Ethiopia

METHODS: A cross-sectional study was conducted at the University of Gondar Hospital from February to March 2016. All the required data were collected using established quality indicators. Data were analyzed using SPSS version 20. Frequencies and cross-tabulations were used to summarize descriptive statistics.

RESULTS: A total of 3259 samples and corresponding laboratory request forms were received for analysis. The analysis of the overall distribution of errors revealed that $89.6 \%$ were preanalytical errors, $2.6 \%$ were analytical, and $7.7 \%$ were postanalytical errors. Of the pre-analytical errors, incomplete request form filling was the most frequent error observed, followed by sample rejection rate (3.8\%). Analytical errors related to internal and external quality control exceeding the target range, (14.4\%) and (51.4\%) respectively, were reported. Excessive turnaround time and unreported critical value cases were the major defects in the post-analytical phase of quality assurance.

CONCLUSION: The present finding showed relatively high frequency of errors, which alarms the importance of quality indicators to assess errors in the total testing process. The University of Gondar Hospital laboratory should improve the quality of healthcare services based on these findings using laboratory standards.

KEYWORDS: Analytical errors; clinical laboratory; postanalytical errors; pre-analytical errors; quality 


\section{INTRODUCTION}

Laboratory services are the backbone of the modern healthcare sector (1). Diagnostic test results provide information that can be used to aid the patient, the physician and others in reaching decisions (2). The impact of laboratory testing in patient care contributes to greater than $60 \%$ of medical decisions (3). However, the analysis performed in the laboratory is subjected to variance; hence, every clinical laboratory must have adequate procedures to assure the quality of the medical results reported. The quality of tests performed in laboratory medicine must allow clinicians to practice good medicine (4-6).

Quality in laboratory medicine is the guarantee that each and every step in the total testing process (TTP) is correctly performed, thus ensuring valuable decision making and effective patient care (6). Errors can occur in any step of the process. These in turn directly lead to increased healthcare costs and decreased patient satisfaction. The impact of error during laboratory testing process can affect patient care in many ways, such as delay in reporting, unnecessary redraws, misdiagnosis, and improper treatment (7).

Evidence in recent decades proves that pre-, intra- and post-analytical processes are equally important for ensuring quality laboratory service. Thus, quality in clinical laboratories should be assured by focusing on all analytical aspects. Studies showed that the pre-analytical phase accounts for $46 \%$ to $68.2 \%$ of the errors observed during the TTP compared to $13-32 \%$ in the analytical and $19-47 \%$ in the post-analytical phases. Pre-analytical and post-analytical errors combined account for $93 \%$ of the total errors encountered in the laboratory (8-10).

Evidence showed that the risk of inappropriate care due to laboratory errors ranges from $6.4 \%$ to $12 \%$ and the incidence of further inappropriate investigations is much higher (19\%) $(11,12)$. Poor laboratory performance that causes an error and delays in diagnosis, and treatment is an obstacle to optimal patient care, particularly in high volume patient care areas such as the
University of Gondar (UOG) Hospital. Although a study was conducted in UOG Hospital laboratory focused on pre-analytical errors only, it did not show errors occurring during the TTP. In addition, UOG Hospital laboratory was recognized as a three-star level laboratory in the World Health Organization Regional Office for Africa accreditation system on November 18, 2011, but its level has come down to one-star level on September 1, 2015 (13). That is a big issue which questions laboratory performance.

Identifying and evaluating errors in TTP is mandatory by using a quality indicator (14). The hypothesis of this study was an inspection of the TTP using quality indicator would enable identification of critical errors at any point of laboratory procedure. In addition, persistent evidence-based identification of laboratory performance through evaluation of pre-, intra-, and post-analytical errors that undermine laboratory quality status helps to put a new strategy to minimize and control errors, and improve processes. Therefore, the aim of this study was to assess errors in TTP in the Clinical Chemistry laboratory of UOG Hospital.

\section{METHODS AND MATERIALS}

Study area: The study was conducted at UOG Hospital laboratory. Gondar Town is located 738 $\mathrm{km}$ far from Addis Ababa, the capital city of Ethiopia. The town has around nine government health centers and one University Hospital. The Hospital is a tertiary level teaching Hospital that provides health services for more than 5 million inhabitants in Northwest Ethiopia. UOG Hospital has diagnostic laboratory service like Clinical Chemistry, Hematology, Microbiology, Parasitology, Serology, Urinalysis and Emergency laboratories that plays important role in teaching, research and community service. The laboratory is equipped with automated instruments in the Clinical Chemistry section, such as Mindray - BS 120 Auto Chemistry Analyzer, mini $V I D A S^{\circledR}$ automated immunoassay, and Roche Diagnostics AVL 9180 Series Electrolyte Analyzers. Previously, there was laboratory information system (LIS). However, the LIS

DOI: http://dx.doi.org/10.4314/ejhs.v28i2.15 
service currently is non-functional during the study period. Clinical Chemistry performs a large number of tests. These include lipid profile tests, kidney function tests, liver function tests, electrolyte profile tests, hormonal analysis, cancer markers detection, measurement of glucose and troponin. Approximately on average, 120 samples are run per day. These tests are manipulated in Clinical Chemistry with four medical laboratory scientists and two clinical chemists.

Study design and period: A cross-sectional study was conducted from February 1 to March 30,2016 , to assess errors in TTP in the Clinical Chemistry laboratory of UOG Hospital.

Sample size and sampling technique: The study included all test requests that were referred for Clinical Chemistry tests at the UOG Hospital during the study period. The total sample size of the study was the total number of requests ordered for Clinical Chemistry tests during the study period. All test requests ordered for Clinical Chemistry tests taking into account venous blood sample were included using consecutive sampling technique.

Data collection methods and processes: Process inspection sheets were formulated to help in the evaluation of pre-, intra- and post-analytical errors for Clinical Chemistry tests at UOG Hospital. Inspection sheets were based upon the International Federation of Clinical Chemistry (IFCC) approved quality indicators (14) and literature review of similar studies $(9,15-18)$ (Table 1). Nine investigators participated in this study. Training was given for all investigators. Data were collected in the Clinical Chemistry section during routine hours each day in the study period. The principal investigator has closely followed and checked the data collection process to ensure the completeness and consistency of the collected data.

Inclusion and exclusion criteria: The study was conducted on processes at pre-, intra- and postanalytical phases. Requests which were ordered for Clinical Chemistry tests taking into account venous blood sample (serum sample) were included in the study.

Urine and other body fluids such as serous fluid, synovial fluid and cerebrospinal fluid were not included in this study. Moreover, test requests ordered for Clinical Chemistry tests only were included. Those requested analyses for Hematology, Coagulation, Serology, Urinalysis, Emergency laboratory and Microbiology were not included in this study.

Data management and quality control: Pre-test of the checklist was done to check clarity, acceptability, and consistency of the structured inspection sheets. The necessary correction was taken before the actual data collected. The data collection, in accordance with quality indicators, was checked by a senior laboratory technologist and the principal investigator. Close follow-up was done by the principal investigator. The filled checklist was collected after consistency and completeness were checked.

Data analysis and interpretation: The data were checked for completeness and entered to EPI info version 3.5.3 and transferred to Statistical Package for the Social Sciences (SPSS) version 20 (IBM Corporation, New York, United States) for analysis. Frequencies, and cross tabulations were used to summarize descriptive statistics. The data were compared using independent t-test.

Ethical clearance: Ethical clearance was taken from the Research and Ethical Review Committee of School of Biomedical and Laboratory Sciences, UOG. Permission letter was secured from both the medical director of the hospital and the diagnostic coordinator of UOG hospital. All the collected data were kept confidential by using codes instead of any personal identifiers. Detectable errors were linked to the responsible personnel for better patient management and quality improvement purpose.

\section{RESULTS}

According to this study, 3259 blood samples and their request papers were inspected. Of these, $2287(70.1 \%)$ were from the Outpatient Department (OPD) and 972(29.8\%) were from the Inpatient Department (IPD).

Parameters on the test request form: The information provided on each request form was examined. Only $3(0.09 \%)$ requisition papers were found to have complete data (a requested paper containing all the necessary information); the rest

DOI: http://dx.doi.org/10.4314/ejhs.v28i2.15 
did not contain full information that they were supposed to contain. Indicators of patient information such as clinical data 3226(99\%) were not filled on the test request form. However, patient's name, sex, age and hospital number were relatively more mentioned. The name,
1262(38.7\%), and the exact address, 60(1.8\%), of the requesting physicians were not specified on the test request form. Sample quality indicators on test request form, time of collection $(99.3 \%)$ and date of sampling $(40.1 \%)$ were missed on test request form (Table 2 ).

Table 1: Quality indicators in the pre-analytical, analytical and post-analytical phases

\section{Quality indicators of pre-analytical phase}

Number of requests with errors concerning patient name/total number of requests

Number of requests with errors concerning hospital number/total number of requests

Number of requests with errors concerning patient sex/total number of requests

Number of requests with errors concerning patient age/total number of requests

Number of requests with errors concerning physician name/total number of requests

Number of requests with errors concerning patient clinical data/total number of requests

Number of requests with errors concerning patient location/total number of requests

Number of requests with errors concerning date of sampling/total number of requests

Number of requests with errors concerning test ordered/total number of requests

Number of requests with errors concerning time of sampling/total number of requests

Number of samples haemolysed/total number of samples

Number of samples lipemic /total number of samples

Number of insufficient volume of samples/ total number of samples

Number of samples mislabeled /total number of samples

Number of samples with no request/total number of samples

Quality indicators of analytical phase

Number of IQC values that exceed the selected target/total quality control run

Number of EQC exceeding the target range in EQAS-PT schemes / total number of quality control runin EQA schemes

\section{Quality indicators of post-analytical phase}

Number of reports delivered outside the specified time/total number of reports

Number of critical values not communicated/total number of reports

Number of data transcription errors/total number of reports

EQAS: External Quality Assessment schemes,EQC: external quality control, IQC: Internal Quality Control, IPD: Inpatient Department, OPD: Out Patient Department, PT: Proficiency Testing, UOG: University of Gondar

Table 2: Frequency of missed data on routinely submitted test request forms in Clinical Chemistry laboratory at UOG Hospital from February to March 2016, Northwest Ethiopia

\begin{tabular}{lllll}
\hline Data type & OPD N(\%) & IPD N $(\%)^{\mathrm{n}=972}$ & ${\text { Total N }(\%)^{\mathrm{n}=3259}}$ & Sig. (2-tailed) \\
\hline Patient name & $0(0.0)$ & $0(0.0)$ & $0(0.0)$ & \\
Hospital number & $1(0.04)$ & $3(0.3)$ & $4(0.1)$ & 0.048 \\
Patient sex & $13(0.6)$ & $5(0.5)$ & $18(0.6)$ & 0.849 \\
Patient age & $14(0.6)$ & $5(0.5)$ & $19(0.6)$ & 0.737 \\
Physician name & $1006(44.0)$ & $256(26.3)$ & $1262(38.7)$ & 0.000 \\
Patient clinical data & $2269(99.2)$ & $957(98.5)$ & $3226(99.0)$ & 0.049 \\
Patient location & $32(1.4)$ & $28(2.9)$ & $60(1.8)$ & 0.000 \\
Date of sampling & $1072(46.9)$ & $235(24.2)$ & $1307(40.1)$ & 0.000 \\
Test ordered & $1(0.04)$ & $0(0.0)$ & $1(0.03)$ & 0.515 \\
Time of sampling & $2281(99.7)$ & $956(98.4)$ & $3237(99.3)$ & 0.000 \\
Over all incomplete request form & $2286(99.9)$ & $970(99.8)$ & $3256(99.9)$ & 0.160 \\
\hline
\end{tabular}

DOI: http://dx.doi.org/10.4314/ejhs.v28i2.15 
Sample quality indicators: The other observation made during the study period was an assessment of sample quality. Of the total number of 3259 samples submitted to the laboratory for Clinical Chemistry tests, $123(3.8 \%)$ were rejected. The most common reason for sample rejection was haemolysis, 41(1.3\%), followed by the request with no sample or sample with no request, 39(1.2\%), and mislabeled, 35(1.1\%) (Table 3).

Quality indicators covering the analytical phase: Unacceptable performances in external quality control (EQC) accounted for 18(51.4\%), followed by non-conformity of internal quality control (IQC) 81(14.4\%) (Table 4).

Table 3: Type and frequency of reason for sample rejected in Clinical Chemistry laboratory at UOG Hospital from February to March 2016, Northwest Ethiopia.

\begin{tabular}{llll}
\hline Parameter & OPD & IPD & Total \\
& $\mathrm{N}(\%)$ & $\mathrm{N}(\%)$ & $\mathrm{N}(\%)^{\mathrm{n}=3259}$ \\
\hline Haemolysis & $19(0.6)$ & $22(0.7)$ & $41(1.3)$ \\
Lipemic sample & $4(0.1)$ & $0(0.0)$ & $4(0.1)$ \\
Insufficient volume of sample & $0(0.0)$ & $2(0.1)$ & $2(0.1)$ \\
Mislabeled & $29(0.9)$ & $6(0.2)$ & $35(1.1)$ \\
Sample with no request/request with no & $28(0.9)$ & $11(0.3)$ & $39(1.2)$ \\
sample & & & \\
Test not ordered/inappropriate test & $0(0.0)$ & $2(0.1)$ & $2(0.1)$ \\
Total & $80(2.5)$ & $43(1.3)$ & $123(3.8)$ \\
\hline
\end{tabular}

IPD: Inpatient Department, OPD: Out Patient Department, UOG: University of Gondar

Table 4: Error frequency of analytical phase in Clinical Chemistry laboratory at UOG Hospital from February to March 2016, Northwest Ethiopia.

\begin{tabular}{llll}
\hline & IQC N (\%) & EQC N (\%) & Total N (\%) \\
\hline Pass & $481(85.6)$ & $17(48.6)$ & $498(83.4)$ \\
Fail & $81(14.4)$ & $18(51.4)$ & $99(16.6)$ \\
Total & $562(100.0)$ & $35(100.0)$ & $597(100.0)$ \\
\hline
\end{tabular}

IQC: Internal Quality Control, EQC: External Quality Control

Quality indicators in the post-analytical phase: In the post-analytical phase, 291(9.3\%) errors were observed. Excessive turnaround time (TAT), $270(8.6 \%)$, contributed to the majority of postanalytical errors followed by unreported critical value cases, 15(0.48). All critical value cases were not communicated to the concerned physician. Although this laboratory does not have functional laboratory information system (LIS) currently, manual reporting accounted for only $6(0.2 \%)$ transcription errors (Table 5).

Pre-analytical phase was found to be error prone process among the total errors (Table 6).

DOI: http://dx.doi.org/10.4314/ejhs.v28i2.15 
Table 5: Error frequency of post-analytical phase in Clinical Chemistry laboratory at UOG Hospital from February to March 2016, Northwest Ethiopia.

\begin{tabular}{ll}
\hline Parameter & \multicolumn{1}{c}{$(\mathrm{n}=3136)$} \\
& \multicolumn{1}{c}{ Frequency (\%) } \\
\hline Excessive TAT & $270(8.6)$ \\
Not informed critical result & $15(0.48)$ \\
Data transcription errors & $6(0.2)$ \\
Total & $291(9.3)$ \\
\hline
\end{tabular}

TAT: turnaround time, UOG: University of Gondar

Table 6: Distribution of total testing process error frequency in the Clinical Chemistry laboratory at UOG Hospital from February to March 2016, Northwest Ethiopia.

\begin{tabular}{lll}
\hline Phase & Error frequency $(\%)$ & Error frequency $(\%)$ \\
\hline & A & B \\
\hline Pre-analytical*6518 $^{*}$ & $3379(51.8)$ & $3379(89.6)$ \\
Analytical $^{* 597}$ & $99(16.6)$ & $99(2.6)$ \\
Post-analytical $^{3136}$ & $291(9.3)$ & $291(7.7)$ \\
Total $^{10251}$ & $3769(36.8)$ & $3769(100)$ \\
\hline
\end{tabular}

The asterisk $(*)$ indicates denominator for each phase in the ' $\mathrm{A}$ ' column, A: Error frequency in the pre-analytical, analytical, and post-analytical phases, B: Overall percent of errors in the three analytical phases

\section{DISCUSSION}

In this study, errors in TTP in Clinical Chemistry laboratory was assessed based on IFCC quality indicators. Currently, there is considerable emphasis on managing TTP in clinical laboratories. This supports a quantitative basis for interested parties aiming to guarantee improvement and up-to-date performance in care and processes $(14,19)$.

Accordingly, 3259 request papers submitted to the Clinical Chemistry laboratory, preanalytical errors associated with request form accounted for $49.9 \%$ due to the omission of important data. Significantly, 3256(99.9\%) request papers were incomplete lacking one or more of the required information. The only welldocumented parameter appeared on all request forms in this study was the patient's name. This result was in line with findings from Ethiopia (18), Pakistan (20) and Ghana (21). This was not astonishing since it was very likely that the request would have been rejected if the patient's name was not mentioned. However, the name of the attending physician was omitted in $38.7 \%$ of test request forms observed. This figure was higher than a study conducted in Nigeria which reported $19.8 \%(22)$. The rationale behind this figure could be attributed to lack of awareness and variability of physicians attending the patient on one site since most physicians visiting the patient were interns.

Besides, this study showed that clinical data (99\%) and time of sampling (99.3\%) were found to be incomplete on the request forms. This result was consistent with a previous study done in a similar setting in Ethiopia (18) in which the authors stated that clinical data $(97.8 \%)$ and time of sampling (100\%) had been missed. This result indicated no improvement after the previous report at similar setting in Ethiopia (18) which demands extra management effort to create awareness concerning the impact of incomplete test request form on the quality of patient care.

DOI: http://dx.doi.org/10.4314/ejhs.v28i2.15 
Moreover, $40.1 \%$ of the request forms observed did not state the date of sampling. This was comparable to results obtained in a similar study conducted in Ghana (37.3\%) (21), but, higher than results obtained in Nigeria $(0.5 \%)$ (23). This variation could be attributed to the workload on physicians, attitudinal difference and negligence among physicians, lack of monitoring by the concerned body or improper orientation about the impact of incomplete test request form on the quality of patient care.

In our study, 3.8\% samples were rejected for various reasons. This figure was lower than a study conducted in India (4.91\%) (19), but higher than the rejection rate reported in Ethiopia (2.4\%) (18), (1.4\%) (24), Turkey (0.91\%) (25) and USA $(0.74 \%)(26)$. High staff turnover, the periodic influx of students, frequent job rotation of phlebotomists, increased patient flow or poor quality management system, would be the main cause of increased sample rejection.

In this study, the most common frequent cause of sample rejection was haemolysis (33.3\%) which is comparable in a study conducted in Ethiopia (24), Nigeria (27) and Spain (28) which reported haemolysis as the main cause of sample rejection. Increased haemolysis observed from this study could be as a result of poor phlebotomy procedures or periodic influx of students in the institutions.

Remarkably, the second frequent reason for sample rejection was a sample with no request/request with no sample accounted for $31.7 \%$, which is higher than the result reported in Ethiopia (24). The same figure for mislabeled samples was $28.5 \%$ in the current study. This could be due to excessive patient load (disproportionate of patients to phlebotomists), the absence of functional LIS and pneumatic tube complicated proper labeling and delivery of samples with corresponding request forms. Loss of attention and poor communication between staffs might further aggravate the problem.

In our study, a total of $16.6 \%$ analytical errors were seen as compared to an Indian report (5.07\%) (19). Of these, unacceptable performance in IQC accounted for $14.4 \%$, which could be due to the improper reconstitution of quality control
(QC) material, inappropriate storage, instability of reagents or contamination or calibration drift. This figure $(14.4 \%)$ was much higher than an Indian report $(0.6 \%)$ of non-conformity of QC (29). The difference could be attributed to the difference in the use of QC material, operator, type of machine, environmental condition, or implementation of quality assurance system.

Unsatisfactory evidence has been found from the external agency as part of the involvement of proficiency testing program which showed EQC exceeding the target range accounted for $51.4 \%$, indicated a need to address shortcomings related to analytical process, which is much higher as compared to a report of Spain (0.8\%) (30). Instability of the instrument due to fluctuation in electricity, lack of laboratory staff training about automation and quality management system, staff turnover or frequent changes of staffs without training could be attributed to the remarkable increment in the analytical errors.

In the current study, the frequency of errors in post-analytical phase was $9.3 \%$, almost triple than the study conducted in India (3.2\%) (29). Even though, the percentage of transcription error contributes much in some literature (29), in this study, excessive TAT $(8.6 \%)$ contributed to the majority of post-analytical errors. Electrical fluctuation, shortage of distilled water and workload could be the cause of not reporting results within a specified time. Manual reporting of results accounted only $0.2 \%$ transcription errors. Since it is all about the life of the human being, it does not mean that the result of this study is low.

Another important aspect of the postanalytical phase of the testing process was critical value reporting. On examination of critical value reporting, $15(0.48 \%)$ critical value cases were observed almost double in the Indian report $(0.21 \%)$ (19), and none of them has been communicated to the concerned physician. Lack of functional LIS, poor awareness among laboratory staffs, missed parameter on the test request form such as patient address, attending physicians and telephone complicated in difficulty to notify within the target time. Failure to deliver a critical value notification within the indicated 
time could be life-threatening if the patient is left untreated. In fact, implementation of electronic LIS can improve the post-analytical phase. This might eliminate transcriptional errors and delay in results.

In general, the overall statistics showed that the error frequency was $89.6 \%$ in the preanalytical phase, $2.6 \%$ in the analytical and $7.7 \%$ in the post-analytical phase. Results reported in the Netherlands with a certain difference to this study showed that the distribution of errors; preanalytical $68.2 \%$, analytical $13.3 \%$ and postanalytical $18.5 \%$ (31). This variation is difficult to explain with regard to the relative frequency of errors observed in the different phases, being different in work complexity, in the implementation of quality management system and method of error detection. Similarly, the frequency of errors may differ from institution to institution and time to time.

This study provides literature regarding the errors in the total testing process in clinical laboratories and points out errors in the TTP that affect the quality of the laboratory service. Therefore, errors detected can be prevented from recurring, thus; make better laboratory quality. As a limitation to this study, the whole story of errors in TTP can not be addressed. Hence, further indepth study to evaluate details of errors in TTP, including pre-pre-analytical phases such as sampling, sample transportation and the like, and post-post-analytical phases should be conducted. Another limitation was the inability to assess the impacts of laboratory errors on patient health and the entire healthcare system. These can be a possible area for future research.

In conclusion, this study reported a high frequency of errors in the TTP. These indicate a need to address shortcomings related to each analytical process. Therefore, a continuous practice of assessing errors is mandatory to help in devising corrective strategies. This helps to improve laboratory performance and hence effective clinical decision-making process.

\section{ACKNOWLEDGEMENT}

We are very grateful to the University of Gondar, College of Medicine and Health Sciences, School of Biomedical and Laboratory Sciences, for logistic and financial support for this study. Our special thanks also go to the University of Gondar Hospital Laboratory staff members, especially Clinical Chemistry Laboratory workers for their kind cooperation during data collection.

\section{REFERENCES}

1. McClatchey KD. Clinical laboratory medicine: Lippincott Williams \& Wilkins; 2002.

2. Armbruster D, Miller RR. The Joint Committee for Traceability in Laboratory Medicine (JCTLM): a global approach to promote the standardisation of clinical laboratory test results. Clinical Biochemist Reviews 2007; 28(3):105.

3. Forsman RW. Why is the laboratory an afterthought for managed care organizations? Clinical Chemistry 1996; 42(5):813-6.

4. Guder WG, Narayanan S, Wisser H, Zawta B. Diagnostic samples: from the patient to the laboratory: the impact of preanalytical variables on the quality of laboratory results: John Wiley \& Sons; 2014.

5. Fraser CG. Biological variation: from principles to practice: Amer. Assoc. for Clinical Chemistry; 2001.

6. Trivedi P, Shah N, Ramani K. Managing Clinical laboratories: Monitor and Control lab errors to improve Lab Performance: Indian Institute of Management; 2011.

7. Kaushik N, Green S. Pre-analytical errors: their impact and how to minimize them. Medical Laboratory Observer 2014; 46(5):22-6.

8. Vidriales M, Clar M, Lecha HD, Fernández M, Vizcaíno S. Errores relacionados con el laboratorio clínico. Química Clínica 2007; 26(1):23-8.

9. Plebani M. Errors in clinical laboratories or errors in laboratory medicine? Clinical Chemical Laboratory Medicine 2006; 44(6):750-9. 
10. Bonini P, Plebani M, Ceriotti F, Rubboli F. Errors in laboratory medicine. Clinical chemistry 2002; 48(5):691-8.

11. Lippi G, Simundic A-M, Mattiuzzi C. Overview on patient safety in healthcare and laboratory diagnostics. Biochemia Medica 2010; 20(2):131-42.

12. Plebani M, Carraro P. Mistakes in a stat laboratory: types and frequency. Clinical Chemistry 1997; 43(8):1348-51.

13. Gershy-Damet G-M, Rotz P, Cross D, Cham F, Ndihokubwayo J-B, Fine G, et al. The World Health Organization African Region Laboratory Accreditation Process. American journal of clinical pathology 2010; 134(3):393-400.

14. Plebani M, Astion ML, Barth JH, Chen W, de Oliveira Galoro CA, Escuer MI, et al. Harmonization of quality indicators in laboratory medicine. A preliminary consensus. Clinical Chemistry and Laboratory Medicine (CCLM) 2014; 52(7):951-8.

15. Plebani M, Sciacovelli L, Marinova M, Marcuccitti J, Chiozza ML. Quality indicators in laboratory medicine: A fundamental tool for quality and patient safety. Clinical biochemistry 2013; 46(13):1170-4.

16. Hawkins R. Managing the pre-and postanalytical phases of the total testing process. Ann Lab Med 2012; 32(1):5-16.

17. Ashakiran S, Sumati M, Murthy NK. A study of pre-analytical variables in clinical biochemistry laboratory. Clinical biochemistry 2011; 44(10):944-5.

18. Addis $\mathrm{Z}$, Wondimagegn $\mathrm{T}$, Tachebele $\mathrm{B}$. Types and frequency of pre-analytical errors at University of Gondar hospital laboratory. Elective Medicine Journal 2015; 2(4):363-5.

19. Agarwal R, Chaturvedi S, Chhillar N, Goyal R, Pant I, Tripathi CB. Role of intervention on laboratory performance: Evaluation of quality indicators in a Tertiary Care Hospital. Indian Journal of Clinical Biochemistry 2012; 27(1):61-8.

20. Malik MF, Khan DA, Ansari WM, Khan FA. Injudicious use of laboratory facilities in tertiary care hospitals at rawalpindi, pakistan: a cross-sectional descriptive study. $B M C$ health services research 2013; 13(1):1.

21. Olayemi E, Asiamah-Broni R. Evaluation of request forms submitted to the haematology laboratory in a Ghanaian tertiary hospital. Pan African Medical Journal 2011; 8(1).

22. Oladeinde BH, Omoregie R, Osakue EO, Onifade AA. Evaluation of laboratory request forms for incomplete data at a rural tertiary hospital in Nigeria. New Zealand Journal of Medical Laboratory Science 2012; 66(2):39.

23. Jegede F, Mbah HA, Dakata A, Gwarzo DH, Abdulrahman SA, Kuliya-Gwarzo A. Evaluating laboratory request forms submitted to haematology and blood transfusion departments at a hospital in Northwest Nigeria. African Journal of Laboratory Medicine 2016; 5(1):6 pages.

24. Molla H, Tegaye A, Hassen F. Frequency of Specimen Rejection and Associated Factors at St. Paul Hospital Millennium Medical College, Addis Ababa.Journal of Multidisciplinary Research in Healthcare 2015; 2(1): 1-16.

25. Atay A, Demir L, Cuhadar S, Saglam G, Unal $\mathrm{H}$, Aksun $\mathrm{S}$, et al. Clinical biochemistry laboratory rejection rates due to various types of preanalytical errors. Biochemia medica 2014; 24(3):376-82.

26. Stark A, Jones BA, Chapman D, Well K, Krajenta R, Meier FA, et al. Clinical laboratory specimen rejection-association with the site of patient care and patients' characteristics: findings from a single health care organization. Archives of pathology \& laboratory medicine 2007; 131(4):588-92.

27. Chawla R, Goswami B, Singh B, Chawla A, Gupta VK, Mallika V. Evaluating laboratory performance with quality indicators. Laboratory Medicine 2010; 41(5):297-300.

28. Ricós C, García-Victoria M. Quality indicators and specifications for the extraanalytical phases in clinical laboratory management. Clinical chemistry and laboratory medicine 2004; 42(6):578-82.

29. Goswami B, Singh B, Chawla R, Mallika V. Evaluation of errors in a clinical laboratory: a 
one-year experience. Clinical Chemistry and Laboratory Medicine 2010; 48(1):63-6.

30. Alsina Kirchner MJ, Funes VA, Adzet CB, Clar M, Escuer MI, Girona JM, et al. Quality indicators and specifications for key processes in clinical laboratories: a preliminary experience. Clinical Chemical Laboratory Medicine 2007; 45(5):672-7.

31. Carraro P, Plebani M. Errors in a stat laboratory: types and frequencies 10 years later. Clinical chemistry 2007; 53(7):1338-42. 